\title{
Política de equidad en el acceso a la información: avanzando hacia un Chile digital
}

\author{
Clara Budnik Sinay \\ Bibliotecaria de la Universidad de Chile. Fue Directora del Programa \\ de Documentación e Información del Banco del Libro, Caracas - \\ Venezuela. Subdirectora de la Biblioteca Nacional de Chile. \\ Actualmente es la directora ejecutiva de la Fundación Democracia y \\ Desarrollo. \\ E-mail: cbudnik@fdd.cl
}

\author{
María Luisa de la Maza Michelson \\ Ingeniero civil industrial de la Universidad de Chile, MBA de ESADE, \\ España. Desde agosto del año 2002 a la fecha se ha desempeñado como \\ coordinadora nacional de BiblioRedes, en la Dirección de Bibliotecas, \\ Archivos y Museos de Chile. \\ E-mail: mlmaza@biblioredes.org
}

\section{Resumen}

El mundo digital rompe completamente con la secuencia y linealidad característica en la lectura del texto impreso, incorporando además sonidos e imágenes. Chile, no ha quedado ajeno a este proceso de cambios. Es así como en el año 98' se inició con el trabajo de un grupo de expertos, la definición de políticas públicas asociadas con las nuevas tecnologías a objeto de impulsar la universalización del acceso, el desarrollo de nuevas capacidades competitivas y la modernización del Estado. Otro hito importante fue la elaboración de la denominada Agenda Digital que también fijó medidas concretas en esta materia para el bienio 20042006. Dentro de estos documentos, queremos destacar en especial la política de equidad en el acceso a la información. En la ejecución práctica se cita el caso BiblioRedes, programa que ofrece internet y capacitación gratis en 378 bibliotecas públicas distribuidas en todo Chile, cuyos resultados han sido reconocidos internacionalmente.

\section{Palabras claves}

Acceso libre a la información. Sociedad de la información. Políticas públicas de información. Agenda digital. Chile, BiblioRedes. Internet. Alfabetización digital. Lectura. Bibliotecas públicas.

\section{Política de eqüidade no acesso á informação: avançando rumo a um Chile digital}

\section{Resumo}

O mundo digital rompe completamente com a seqüência e linearidade, características da leitura do texto impresso, incorporando, além disso, sons e imagens. O Chile não ficou alheio a este processo de mudanças. Tanto assim que, no ano de 1998, um grupo de especialistas iniciou um trabalho para definição de políticas públicas associadas às novas tecnologias, com o objetivo de impulsionar a universalização de acesso ao desenvolvimento de novas capacidades competitivas e de modernização do Estado. Outro marco importante foi a elaboração da chamada Agenda Digital, que também fixou medidas concretas sobre o assunto, para 0 biênio 2004-2006. Entre estes documentos destacamos, em especial, a política de eqüidade no acesso à informação. $\mathrm{Na}$ execução prática é citado o caso da BiblioRedes, programa que oferece acesso à Internet e capacitação gratuita na rede, em 378 bibliotecas públicas distribuídas por todo o Chile, cujos resultados têm sido reconhecidos internacionalmente.

\section{Palavras-chave}

Acesso livre à informação. Sociedade da informação. Políticas públicas de informação. Agenda digital. Chile, BiblioRedes. Internet. Alfabetização digital. Leitura. Bibliotecas públicas.

\section{Free access policy for information access: towards a digital Chile}

\begin{abstract}
The digital world breaks two characteristics of the printed text - sequence and linearity; it also supports sounds and images. Chile has not been apart from this evolution. In 1998, a team of experts started to work towards the definition of public policies focusing widespread access, new competitive capabilities and the modernization of the public administration. Another important measure was the launching of the Digital Agenda, a document addressing practical actions for the period $2004-2006$. The policy of equal rights to information access is a very important conceptual result amongst the many documents. Biblio Redes is a practical implementation - it offers Internet access and training in 387 public libraries all over Chile. Its results are internationally recognized.
\end{abstract}

\section{Keywords}

Free access to information. Information society. Public information policies. Digital agenda. Chile, BiblioNetworks (BiblioRedes). Internet. Digital literacy. Reading. Public libraries. 


\section{LA LECTURA, EN LA SOCIEDAD DE LA INFORMACIÓN}

Para iniciar cualquier diálogo sobre el acceso a la información, necesariamente debemos hacer una reflexión sobre el mundo de la lectura, ya que ha sido la base para incorporarse a la sociedad de la información. Para una mejor comprensión de los procesos involucrados, analizaremos a continuación algunos de los siguientes elementos que dan cuenta de su complejidad.

Existen diferentes modalidades de lectura, condicionadas por la existencia, más o menos autónoma, pero siempre en una permanente interacción, del mundo del texto y el mundo del lector, enfrentados ambos a sus propios procesos de cambio y transformación.

Luego, que el texto no se reduce a su comprensión semántica y que los diversos lectores y maneras de leer lo recrean, otorgándole significados. Al mismo tiempo, las formas que adoptan los textos no son un elemento neutro. Los objetos escritos, los diferentes soportes y las lecturas que posibilitan, otorgan un nuevo sentido a la lectura, en tanto condicionan el contenido llegando a formar parte de él.

Por último, tendríamos que señalar que la lectura es una experiencia que nunca se verifica en el vacío, sino en un marco histórico y en un espacio determinado, y que se manifiesta a través de gestos y hábitos, en medio de comunidades de lectores. Por tanto, la lectura es una práctica que se inscribe en determinadas formas de sociabilidad y relación social del lector con los demás, e incluso, consigo mismo.

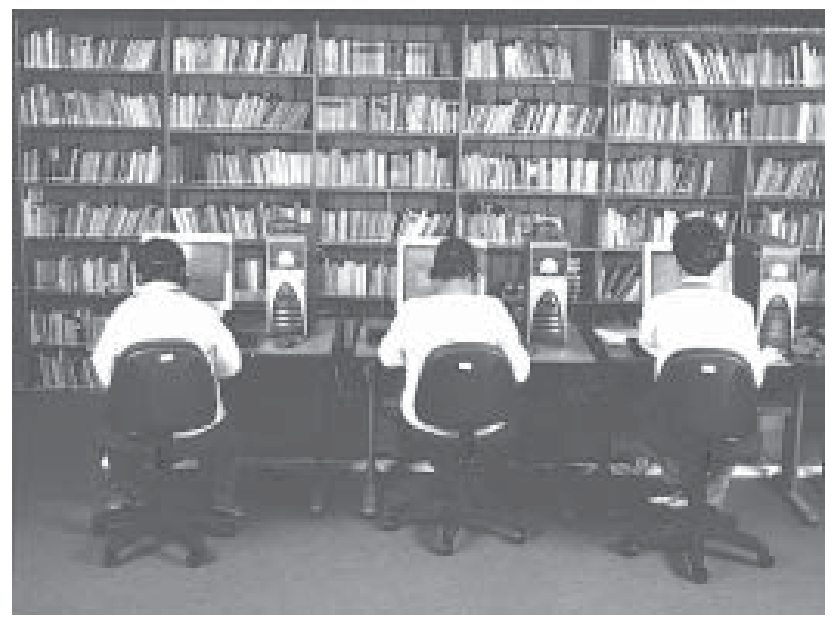

Para llegar a la sociedad de la información, nos remontaremos en la historia, a objeto de comprender a través del tiempo, que la lectura ha ido cumpliendo múltiples funciones sociales asociadas, principalmente, a la conservación y preservación de la tradición y la cultura, a la difusión y transmisión de la información, a la educación, al goce y a la entretención, a la devoción y la práctica religiosa, como un momento de la vida social.

El lugar que comenzó a privilegiarse para la lectura fue la biblioteca. Es así como en la Roma imperial, la biblioteca era un "espacio donde se hacía la vida", y un lugar de esparcimiento culto de la vida urbana, para quienes desearan efectuar allí su lectura.

Varios siglos después, en el siglo XI, la función de las bibliotecas se hizo más compleja, sumando a su carácter de repositorio de obras, el de espacio para la lectura, el cual, al igual que la iglesia, fue consagrado como un lugar de silencio. En este proceso, los catálogos debieron evolucionar desde el mero inventario a la incorporación de instrumentos más eficaces de búsqueda y consulta.

Entre el siglo XVI y el XIX se produjeron varios y profundos procesos de cambios condicionados, en primer término, por la revolución técnica que constituyó la imprenta que amplió y consolidó el cambio producido durante la Edad Media en las prácticas y modelos de lectura, que impulsó la lectura silenciosa.

Hasta el siglo XVI el libro impreso siguió dependiendo del manuscrito, manteniendo así cierta continuidad en los formatos y características de las obras, pero a partir de la segunda mitad del siglo XVIII, con el incremento de la producción de periódicos e impresos que cuadruplicó las cifras con que había comenzado el siglo, se produjo el cambio de lo que se ha denominado la lectura intensiva a la lectura extensiva.

EI siglo XX abre paso, como nunca antes, a una gran dispersión de los modelos de lectura, proceso iniciado ya en el siglo XIX. Junto a la masiva irrupción de nuevos lectores -- principalmente mujeres, obreros y niños - se mantiene la tendencia al incremento de la producción bibliográfica así como a su diversificación. Este proceso modifica los hábitos de lectura iniciando a los lectores en aquella modalidad tan extendida en nuestros tiempos, asociada a la lectura de consumo y entretención, principalmente, periódicos, revistas y libros que se leen de manera parcial, fragmentaria, entrecortada y no necesariamente lineal, de manera análoga al zapping, hábito formado junto con el desarrollo de los medios audiovisuales, en particular, la televisión. 
El zapping puede ser calificado como una forma caótica de lectura, en oposición a la lectura intensiva, lineal y concentrada, y se le puede considerar como un antecedente de la lectura hipertextual, la que presenta tres características principales:

La primera de ellas es que con el hipertexto se modifica completamente la noción de contexto. La contigüidad física, característica de la lectura y de la relación que se establece entre lector y texto, es reemplazada por una contigüidad lógica.

Asimismo, la materialidad de las obras es sustituida por una presencia virtual que puede materializarse a voluntad del lector, mediante una simple orden a la impresora.

Por último, el lector conquista el dominio sobre el texto, estableciéndose una nueva relación que conduce a la más completa reorganización de la escritura y de la lectura. Esta es una de las dimensiones de lo que se ha venido en llamar interactividad. El lector tiene ahora la posibilidad de leer en cualquier momento, y casi en cualquier lugar, una infinidad de textos, anulando las distancias espaciales y temporales, (pasando por alto eso si, la feroz desigualdad en el acceso a estos recursos que ha dado lugar a lo que se conoce como brecha digital). Pero, sobre todo, ahora el lector tiene también la posibilidad inédita de intervenir y modificar el texto.

Si bien la obra escrita siempre permitió desplazarse en múltiples direcciones y asociaciones -ya que no es una entidad cerrada, sino una relación, un centro de innumerables relaciones- el mundo digital rompe completamente, con la secuencia y linealidad básica que caracteriza a casi todo impreso y va más allá, al permitir la múltiple combinación de texto, sonido e imagen.

Así, el texto es hoy cada vez más plural e infinito. Como en aquel cuento de Borges en que la totalidad se nos ofrece a la percepción simultáneamente, sin superposición y sin transparencia, así, la realidad del hipertexto centellea como esa "pequeña esfera tornasolada, de casi intolerable fulgor" que es el Aleph, una descripción que se nos aparece como una maravillosa y sorprendente metáfora de la nueva realidad de la lectura. De igual manera, Borges, el escritor de lo infinito, nos transporta a la vasta Biblioteca de Babel que "cabría en un solo volumen" que tuviera "un número infinito de hojas, infinitamente delgadas, que se desdoblarán en otras análogas". ¿No es esta, acaso, una perfecta descripción del hipertexto?
Actualmente, la lectura está en un proceso de evolución permanente, cuyos alcances y significado continúan desplegándose, lo que recomienda cautela a la hora de hacer predicciones. Habrá que ver los efectos de los cambios descritos sobre las nuevas generaciones. La incorporación del niño a las prácticas cotidianas y masivas de lectura, a partir del siglo XIX, es decir, en una fecha relativamente reciente, contrasta con la rápida alfabetización digital que los niños y jóvenes de hoy están experimentado. Esa permeabilidad, cuyo único límite parece estar dado por la desigualdad en el acceso a las nuevas tecnologías, permite pensar que los cambios en curso seguirán modificando y trastocando, en una dimensión aun insospechada, las formas y modelos conocidos de lectura. Así, el viaje que constituye toda lectura se realiza ahora en tierras no sólo ajenas sino, también, ignotas.

\section{LOS PRIMEROS PASOS HACIA UNA ALFABETIZACIÓN DIGITAL}

A fines de la década de los 90' existía una notoria asimetría entre el estancamiento de los indicadores de lectura y compra de libros y periódicos, y el enorme crecimiento de la oferta disponible de televisión y televisión por cable, de ahí en adelante las políticas públicas de acceso a la información no podrían dejar de lado esta realidad, el acceso a la información debería complementar aquellos medios ya conocidos propios del mundo analógico con aquellos que ofrece el entorno digital.

En este contexto, en Chile, se decidió desarrollar políticas públicas específicas que tuvieran como objetivo lograr garantizar el acceso equitativo a la información a todos los ciudadanos. Para ello se decidió hacer un diagnóstico y un plan de trabajo detallado que permitiera aprovechar las oportunidades que ofrecían las Nuevas Tecnologías de Información y Comunicación (NTICs).

En enero de 1999, se publica el documento "Chile: Hacia la Sociedad de la Información", editado por la "Comisión Presidencial Nuevas Tecnologías de Información y Comunicación" que fuera constituida a mediados del año 1998 por S.E. el Presidente de la República don Eduardo Frei Ruiz-Tagle, dicho texto fue comúnmente conocido como el "Libro Azul". La importancia de este documento fue que reunió a expertos que se desempeñaban en distintas áreas tanto públicas como privadas, para elaborar un Plan de Acciones concretas y plenamente viables en un sentido técnico y financiero, para avanzar hacia la Sociedad de la Información, pero sobre todo, 
elevó este tema antes ausente, al nivel de una política pública. Tal como el mismo libro lo señala, este avance “..encierra promesas de prosperidad material, equidad social, revitalización cultural y desarrollo democrático difícilmente imaginables unos pocos años atrás".

La propuesta de acción se ordenó en torno a tres grandes objetivos: I. Impulsar la Universalización del Acceso, II. Desarrollar nuevas Capacidades Competitivas y III. Modernizar el Estado al Servicio del Ciudadano. Cada uno de estos objetivos tiene líneas de desarrollo y da origen a 61 acciones concretas.

Queremos destacar aquí algunas de ellas que son más pertinentes al tema que nos convoca. La propuesta de un Programa Nacional de Información Pública para las personas, propuesta que busca instalar en todas las comunas del país, Quioscos y telecentros comunitarios Internet. Además del Programa de Digitalización del Patrimonio Histórico y Científico de Chile y el Impulsar las Experiencia de Producción Cultural que Utilizan las NTICs.

Sobretodo, quisiéramos destacar la conclusión que a nuestro juicio es la base de la política pública que está vigente hasta el día de hoy en Chile "... mientras más invierta Chile en formación de recursos humanos, en ciencia y tecnología y en la digitalización de su acervo cultural, mayores serán las capacidades nacionales para maximizar el uso de las NTICs para crear conocimientos, difundir información y generar nuevas innovaciones".

Para agregar algunos elementos de contexto, hasta el año 1998, se observaba un lento avance de la infraestructura de información, no abarcaba más de un 5\% de las empresas y $2 \%$ de los hogares. Salvo experiencias como la Red Enlaces y Reuna2, no se advierte una masificación acelerada en el uso de redes digitales.

El año 2003 se elabora otro documento que contiene la política, la Agenda Digital que surge como resultado de la constitución del Grupo de Acción Digital, presidido por el Coordinador Gubernamental de Tecnologías de Información y Comunicación. Este Grupo estaba conformado por instituciones de gobierno, organizaciones representativas del ámbito empresarial, sector académico y de otros poderes del Estado.

El resultado de este esfuerzo es un amplio acuerdo público-privado sobre una estrategia-país, mirando a la celebración del Bicentenario en 2010, y un Plan de
Acción para el período 2004-2006, que contempla 34 iniciativas. Su objetivo es contribuir al desarrollo de Chile mediante el empleo de las tecnologías de información y comunicación (TICs) para incrementar la competitividad, la igualdad de oportunidades, las libertades individuales, la calidad de vida y la eficiencia y transparencia del sector público, enriqueciendo al mismo tiempo la identidad cultural de la Nación y de sus pueblos originarios.

El contenido de la Agenda Digital coincidió plenamente con los principios establecidos por la Primera Cumbre Mundial de la Sociedad de la Información, realizada en Ginebra en diciembre de 2003 y definió una posición proactiva de Chile con miras a la segunda fase de la misma, que se realizó en Túnez en noviembre de 2005.

Durante el segundo semestre del año 2006 se proyecta la elaboración de la agenda digital 2.0 que actualice en base a los avances logrados a la fecha, el plan de acciones a seguir.

En la actualidad existen varias acciones complementarias con el objeto de otorgar a los ciudadanos un acceso igualitario disminuyendo la llamada brecha digital. Se destaca la Red Enlaces cuya misión ha sido dotar de computadores a las escuelas, alcanzando una cobertura del $94 \%$ del total de niños en educación básica y $87 \%$ en educación media del país, contribuyendo en forma masiva a dar acceso y alfabetización digital al público escolar.

Para los adultos existen varias iniciativas que se han agrupado en lo que se ha llamado la Red Nacional de Infocentros y corresponden según el último catastro realizado el año 2005, a 758 lugares de acceso público a computadores e Internet, distribuidos a lo largo del país. Dentro de estas iniciativas de acceso público destacan:

1. El Programa BiblioRedes de la Dirección de Bibliotecas, Archivos y Museos (DIBAM), con 378 bibliotecas públicas conectadas y que ofrecen Internet y capacitación;

2. La Red Enlaces, del Ministerio de Educación, con 100 infocentros/escuelas que abren sus salas de computación a la comunidad un número de horas a la semana para dar acceso y realizar cursos de alfabetización digital para padres y apoderados;

3. El INJUV con 100 infocentros orientados a los jóvenes (ente 15 y 29 años); 
4. El Sercotec con 100 infocentros orientados a los Micro y pequeños empresarios;

5. Otros 80 infocentros pertenecientes a diversas instituciones tanto públicas como privadas.

El año 2002 según el Censo en Chile se registraba un $20 \%$ de los hogares con computador y un $10 \%$ con acceso a Internet, sin embargo, en los últimos años se ha experimentado un fuerte aumento, llegando el año 2004, según el estudio WIPChile a 35\% los usuarios con acceso a Internet.

\section{BIBLIOTECAS: UN ESPACIO IDEAL PARA EL LIBRE ACCESO}

Con el soporte económico y técnico de la Fundación Bill $\&$ Melinda Gates, y con el alto apoyo del Gobierno de Chile, el año 2002 la DIBAM inició el Proyecto BiblioRedes para instalar computadores e Internet inicialmente en 368 Bibliotecas Públicas a lo largo del país. Los mayores beneficios del Proyecto eran lograr un mayor acceso a la información y con ello, mayores oportunidades, especialmente para las comunas aisladas.

Si bien, ya existían iniciativas orientadas hacia los escolares, PYMES y jóvenes, BiblioRedes priorizó dentro de su público objetivo a la comunidad adulta, especialmente a las mujeres, aunque, el proyecto siempre ha ido en beneficio de toda la comunidad. La particular preocupación por las mujeres se debió a que existían antecedentes de que sólo el 30\% de las mujeres trabaja fuera del hogar y un estudio de base e impacto encargado al CIDE de la Universidad Alberto Hurtado específico nos indicaba que dentro del hogar la persona que tenía menores niveles de conocimiento en TICs resultó ser la madre, con un $76 \%$ que declararon no saber cómo usar los computadores, mientras que sólo un $32,4 \%$ de los hijos mayores presentaba el mismo desconocimiento en materia tecnológica.

El Programa BiblioRedes vino a entregar una serie de servicios a las Bibliotecas, entre los que destacan: asistencia en aspectos como planificación y gestión, soporte técnico remoto y en terreno según necesidad, capacitación permanente al personal de la biblioteca y materiales para la capacitación y difusión.

Los servicios que se entregan a la comunidad en forma gratuita por medio de este Programa son: acceso a Internet, alfabetización digital y cursos más avanzados, soporte para publicación de páginas web, incluyendo la capacitación y el hosting, material y guías rápidas para el autoaprendizaje.

\section{¿POR QUÉ EN LAS BIBLIOTECAS PÚBLICAS?}

Al comenzar a implementar el Proyecto, las Bibliotecas Públicas en Chile no se parecían a las bibliotecas de los países desarrollados, pues en general carecían de infraestructura adecuada, aunque lograban ofrecer buenos servicios. La incorporación de herramientas tecnológicas y capacitación permitió reforzar el sitial de importancia de las bibliotecas entre sus comunidades, constituyéndose en un espacio comunitario reconocido, en el cual se puede acceder a información independientemente del soporte en que ésta se encuentre (impreso o digital). La biblioteca se transforma así en un lugar de participación donde se hace tangible la inclusión digital.

La biblioteca es el reflejo de la comunidad en la que está inserta, es por ello que existen de todos los tamaños, colores y formas, algunas modernas y otras patrimoniales con más de 100 años de historia.

La DIBAM había impulsado en las bibliotecas, desde mediados de los 90', una política de estanterías abiertas, de manera de acercar los libros a los usuarios. Siguiendo esta filosofía, se buscó que los computadores estuvieran cerca de los usuarios en espacios compartidos con los libros y no en recintos cerrados.

En el estudio de base e impacto antes referido se indagó sobre los niveles de confianza que generaban en el público distintas instituciones, entre ellas las bibliotecas públicas, observándose que éstas, contaban con un $80,5 \%$ el año 2003 y un $86,4 \%$ el año 2005 , siendo solo superadas por la confianza que existe respecto de la familia. A nuestro juicio, este es un factor muy relevante que hace que la política pública sea exitosa, ya que a la hora de incorporar a las bibliotecas estos nuevos servicios, se ha mejorado el acceso, pero sobretodo en el caso de la capacitación que requiere de un ambiente de confianza para que ocurra el aprendizaje.

BiblioRedes hoy no sólo ha logrado instalar una red digital, sino que ha potenciado una red humana. Confió en el importante rol que ejercían las personas en sus comunidades y se les enseñó a usar el computador, a navegar por Internet, a diseñar el sitio web de su biblioteca y transmitir estos conocimientos a sus comunidades. El personal de las bibliotecas públicas ha recibido aproximadamente 400 horas de capacitación, a través de los ocho ciclos de capacitación realizados a la fecha. 
Política de equidad en el acceso a la información: avanzando hacia un Chile digital

Desde el año 2002 a la fecha, BiblioRedes ha capacitado a más de 212 mil personas, de las cuales 160 mil fueron alfabetizadas digitalmente y a 40 mil personas se les entregó una capacitación computacional complementaria. Todos ellos son parte de los 420 mil usuarios del Programa que han realizado a la fecha 5,5 millones de sesiones de acceso a Internet y han publicado cerca de 7 mil páginas Web. La creación de sitios web ha sido lo que ha significado un reconocimiento internacional del Programa, pero lo más importante a nuestro juicio es que los usuarios de las bibliotecas han pasado de ser "consumidores de información a creadores de contenidos locales en la web."

Estas cifras grafican el rol protagónico de BiblioRedes y las Bibliotecas Públicas en el esfuerzo país por superar la brecha digital, proceso durante el cual las Bibliotecas se han abierto más aún a las comunidades y han adoptado un rol muy importante en el desarrollo y difusión de la cultura local, a través de Internet.

\section{IMPACTO POSITIVO EN LA LECTURA}

Hoy podemos decir que se ha producido un cambio cultural en la red de Bibliotecas Públicas, que se ha visto reflejado - contradiciendo todas las predicciones - en un incremento en las cifras de lectura y de préstamo de libros a domicilio. Es cierto que en los inicios de BiblioRedes había cierto temor de que la computación e Internet desplazaran a los servicios más tradicionales de la biblioteca. En la práctica ha ocurrido todo lo contrario, ya que con la llegada de las NTICs se ha potenciado la biblioteca pública como un lugar privilegiado de acceso a la información, independiente del medio en la que ésta se encuentre.

El impacto positivo de la incorporación de BiblioRedes queda de manifiesto en el estudio de base e impacto ya referido, ya que al preguntar respecto a los motivos de asistencia a la biblioteca, se desprende que el 18\% de usuarios actuales ha llegado atraído por los computadores y antes no venía a la biblioteca, comprobándose el aumento real en el número de usuarios de la biblioteca. Así mismo, el 52\% de los usuarios declara que ahora combina la revisión y petición de libros, con el uso de computador e Internet.

En el mismo estudio también se puede inferir un mejoramiento en la percepción de la calidad de atención, ya que cerca del $80 \%$ de los usuarios considera que en su Biblioteca Pública hay mejor atención que antes, que la entrega de información es más rápida y que existe una mejor orientación a las peticiones que en el pasado. De la misma manera un $56,5 \%$ de los usuarios opina que ahora hay más personal que antes para atender al público y un $79,9 \%$ considera que en las Bibliotecas Públicas existe personal especializado para apoyar el uso de los computadores e Internet.

El incremento de la lectura, asociado a la presencia de BiblioRedes y otras iniciativas, se puede inferir del aumento considerable experimentado en las cifras de préstamo a domicilio de la Subdirección de Bibliotecas Públicas, desde que se iniciara dicho Proyecto. De hecho mientras en el año 2002, los préstamos a domicilio 1legaron a 1.684.328; el año 2005, alcanzaron los 2.976.948. Es decir, se produjo un aumento de un $76 \%$ en un período de 3 años.

\section{EL CAMINO QUE QUEDA POR RECORRER}

Desde fines de la década de los años 90, se diseñó una política pública de libre acceso a la información y desde ese entonces, se ha avanzado con paso firme en la implementación de la misma. Sin embargo, es importante señalar que aun queda mucho camino por recorrer. Así nos lo corrobora la conclusión a la que llega el Informe sobre Desarrollo Humano en Chile 2006. Las tecnologías: ¿Un salto al futuro?, efectuado por el PNUD que nos señala: "La mitad de los chilenos se sienten excluidos de las tecnologías".

El mencionado informe, entregado por el PNUD a la Excma. Presidenta de la República doña Michelle Bachelet el pasado 30 de junio de 2006, nos señala que muchos chilenos creen que las NTICs ayudarán al país a dar un salto al desarrollo, pero la mitad de ellos piensan que así como se puede reducir las desigualdades, también puede crear brechas de nuevo tipo. En el informe se destaca que entre los adolescentes de 14 a 17 años, un $84 \%$ se siente integrado a las NTICs, constatando que "en su mayoría mujeres, jubilados y personas pertenecientes al grupo socioeconómico bajo de menor nivel educacional están fuera del mundo de las nuevas tecnologías".

Tales indicadores nos confirman que debemos orientar nuestros mayores y mejores esfuerzos a aquellos grupos prioritarios que los ejecutores de las políticas públicas han definido para alcanzar el acceso equitativo a la información de todos los ciudadanos. El Programa BiblioRedes como uno de los ejecutores principales de esta política públicas, asume el compromiso de seguir aportando, con todo su equipo humano, la experiencia adquirida en todos estos años para facilitar el logro de tal objetivo y hacer que este sueño de todos, sea una hermosa 


\section{Clara Budnik Sinay / María Luisa de la Maza Michelson}

realidad, profundizando en el concepto que la biblioteca pública es un lugar privilegiado para el desarrollo de la democracia, acceso equitativo a libros y tecnología, y diversos modos de lectura.

Chile: Hacia la Sociedad de la Información, Enero 1999, Informe al Presidente de la República, Comisión Presidencial Nuevas Tecnologías de Información y Comunicación. Gobierno de Chile. Pág. 15.

\section{REFERÊNCIAS}

ACTUAlmente el Programa BiblioRedes está presente en 378 bibliotecas públicas. Disponível em: < $\underline{\text { http://www.biblioredes.cl/ }}$ bibliored/>. Acesso em: 2006.

CHILE: hacia la sociedad de la información. [Chile: s.n.], 1999. 111 p. Informe al Presidente de la República, Comisión Presidencial Nuevas Tecnologías de Información y Comunicación. Disponível em: $<\underline{\text { http:/ }}$ $\lfloor$ www.unesco.org.uy/informatica/publicaciones/chile.pdf $>$. Acesso em: 2006.

DESARROLLO humano en Chile 2006. Las tecnologías: ¿un salto al futuro? Programa de las Naciones Unidas para el Desarrollo. Disponível em: < http://www.pnud.cl $>$. Acesso em: 2006.

DIRECCIÓN de bibliotecas, archivos y museos, entidad autónoma dependiente del Ministerio de Educación del Gobierno de Chile. Disponível em: < http://www.dibam.cl/>. Acesso em: 2006.

ENLACES, centro de educación y tecnología del Ministerio de Educación. Disponível em: < http://www.enlaces.cl >. Acesso em: 2006.

ESTADÍSTICAS del estudio WIPChile 2004. Disponível em: < http:/ Lwww.wipchile.cl>. Acesso em: 2006.

ESTUDIO de base e impacto realizado por el CIDE, Centro de Investigación y Desarrollo de la Educación, de la Universidad Alberto Hurtado, año 2002-2005. Disponível em: < http://www.cide.cl/ > . Acesso em: 2006.

FUENTE: subdirección de bibliotecas públicas de Chile. Disponível em: <http://www.dibam.cl/bibliotecas_publicas/sitios.htm $>$. Acesso em: 2006.

INSTITUTO NACIONAL DE LA JUVENTUD. Disponível em: $<$ http://www.injuv.gob.cl/index.php $>$. Acesso em: 2006.

PYMES: acrónimo utilizado para identificar a las pequeñas y medianas

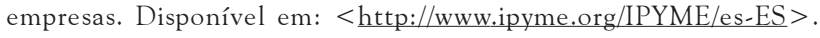
Acesso em: 2006.

RED enlaces: programa de introducción de tecnología en las escuelas, a cargo del Ministerio de Educación. Disponível em: < http:// www.enlaces.cl/>. Acesso em: 2006.

REUNA2: red de alta velocidad para la educación superior chilena. Disponível em: $<$ http://www.reuna.cl/servicios/reuna2.html $>$. Acesso em: 2006.

SERVICIO de cooperación técnica. Disponível em: < http:// www.sercotec.cl/>. Acesso em: 2006. 